\title{
Single top, W and Higgs associated production
}

\author{
Federico Demartin* \\ Centre for Cosmology, Particle Physics and Phenomenology (CP3), \\ Université catholique de Louvain, B-1348 Louvain-la-Neuve, Belgium \\ E-mail: federico.demartin@uclouvain.be
}

\section{Benedikt Maier}

Institut für Experimentelle Kernphysik,

Karlsruher Institut für Technologie (KIT), D-76131 Karlsruhe, Germany

\begin{abstract}
We study the associated production of a single top quark plus a $W$ and a Higgs bosons $(t W H)$ at the LHC, at NLO-QCD accuracy. We show results obtained with the technique known as Diagram Removal to treat the event generation in the NLO real-emission $t W b H$ channel, where $t W H$ interferes with $t t H$. We provide NLO results for $t W H$ total and differential cross sections at $13 \mathrm{TeV}$, also matching the matrix element for the hard scattering to a parton shower. Finally, we show the sensitivity of this process to a generic CP-mixed (i.e. BSM) Yukawa interaction between the Higgs and the top quark.
\end{abstract}

8th International Workshop on Top Quark Physics

14-18 September, 2015

Ischia, Italy

\footnotetext{
* Speaker.
} 


\section{Introduction}

Despite its small cross section at the LHC, $t W H$ production is an interesting case of study for two reasons: (1) rare processes where the Higgs is produced with a single top quark are more sensitive than $t t H$ to study departures of the top Yukawa coupling from the SM prediction (see [1] and references therein), and (2) at NLO-QCD the $t W H$ (singly resonant) and $t t H$ (doubly resonant) processes interfere in the real-emission $t W b H$ channel (fig. 1), thus their separation is not trivial. The same situation is present also in the SM cases of $t W / t t$ production [2,3] and $t W Z / t t Z$, and in beyond-Standard-Model (BSM) processes [4-7]. This problem can be avoided with a full simulation of $W b W b H$ production at NLO [8]; however, this is highly non-trivial. Moreover, efficient generation of singly-resonant events $(t W H)$ require to run separately the $t W H$ and $t t H$ processes. To this purpose, approximate techniques known as Diagram Removal and on-shell Diagram Subtraction are used to treat the $t W b H$ channel. Our results are obtained within the MADGRAPH5_AMC@NLO framework [9].
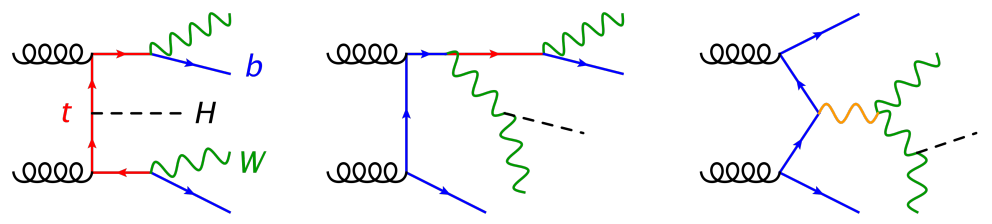

Figure 1: Examples of " $t t H$ " doubly resonant amplitudes $\mathscr{A}_{2}$ (left), " $t W H$ " singly resonant amplitudes $\mathscr{A}_{1}$ (center) and non resonant amplitudes $\mathscr{A}_{0}$ (right) contributing to the same $\mathrm{WbWbH}$ final state.

In the $t W b H$ channel, the Diagram Removal (DR) matrix elements without (DR1) and with interference (DR2) are

$$
\mathscr{M}_{\mathrm{DR} 1}=\left|\mathscr{A}_{1}\right|^{2} \quad \text { and } \quad \mathscr{M}_{\mathrm{DR} 2}=\left|\mathscr{A}_{2}+\mathscr{A}_{1}\right|^{2}-\left|\mathscr{A}_{2}\right|^{2}
$$

where the indices 1 and 2 indicate the number of on-shell top quarks in the diagrams. Both DR1 and DR2 are not guaranteed to preserve gauge invariance. We find a significant difference between DR1 and DR2, because the doubly-singly interference $\mathscr{A}_{2} \mathscr{A}_{1}^{*}$ contribution is large compared to the singly resonant contribution $\left|\mathscr{A}_{1}\right|^{2}$. Non-resonant contributions from $\mathscr{A}_{0}$ (“+0r") are numerically negligible.

We have also investigated results obtained using on-shell Diagram Subtraction (DS)

$$
\mathscr{M}_{\mathrm{DS}}=\left|\mathscr{A}_{2}+\mathscr{A}_{1}\right|^{2}-\mathscr{C}_{\mathrm{DS}} \quad, \quad \mathscr{C}_{\mathrm{DS}}\left(\left\{p_{i}\right\}\right)=\frac{\left(m_{t} \Gamma_{t}\right)^{2}}{\left(p_{b W}^{2}-m_{t}^{2}\right)^{2}+\left(m_{t} \Gamma_{t}\right)^{2}}\left|\mathscr{A}_{2}\left(\left\{q_{i}^{\mathrm{OS}}\right\}\right)\right|^{2},
$$

where $m_{t}$ and $\Gamma_{t}$ are the mass and the width of top quark, while the external momenta reshuffling $\left\{p_{i}\right\} \rightarrow\left\{q_{i}^{\mathrm{OS}}\right\}$ puts on-shell the internal top propagator, to preserve gauge invariance (at least in the limit $\Gamma_{t} / m_{t} \rightarrow 0$ ). We have found a significant dependence on how the on-shell reshuffling $\left\{p_{i}\right\} \rightarrow\left\{q_{i}^{\mathrm{OS}}\right\}$ is performed; further investigations about the substantiality of this issue are ongoing.

\section{2. $t W H$ production at NLO+PS}

The NLO cross section for $t W H$ production at $13 \mathrm{TeV}$, computed with DR2, amounts to $15.6 \mathrm{fb}$ and shows significantly reduced uncertainties compared to LO (table 1). The impact of $t \mathrm{WH} / \mathrm{ttH}$ 
interference can be estimated by taking the difference with the DR1 result ( $\sim 5 \mathrm{fb})$, and amounts to about one third of the NLO cross section. Interference affects significantly also the shape of distributions of the top quark and the $W$ boson, while the effect is milder for the Higgs (fig. 2).

\begin{tabular}{lllll}
\hline$t W H$ & $\sigma[\mathrm{fb}]$ & $\delta_{\mu}^{\%}$ & $\delta_{\mathrm{PDF}}^{\%}$ & $K$ \\
\hline LO & $16.15(3)$ & ${ }_{-12.8}^{+12.9}$ & \pm 11.1 & - \\
NLO DR1 & $20.65(3)$ & ${ }_{-3.2}^{+5.0}$ & \pm 3.0 & 1.28 \\
NLO DR2 & $15.61(3)$ & ${ }_{-6.1}^{+4.6}$ & \pm 2.7 & 0.97 \\
\hline
\end{tabular}

Table 1: Total cross sections at the 13-TeV LHC for $p p \rightarrow t W^{-} H+\bar{t} W^{+} H$, in the $5 \mathrm{~F}$ scheme at LO and NLO accuracy (NLO results are obtained with Diagram Removal). We also report the scale and PDF uncertainties, as well as the NLO $K$ factor. The dynamic scale $\mu=H_{T} / 4$ has been used throughout the simulation.
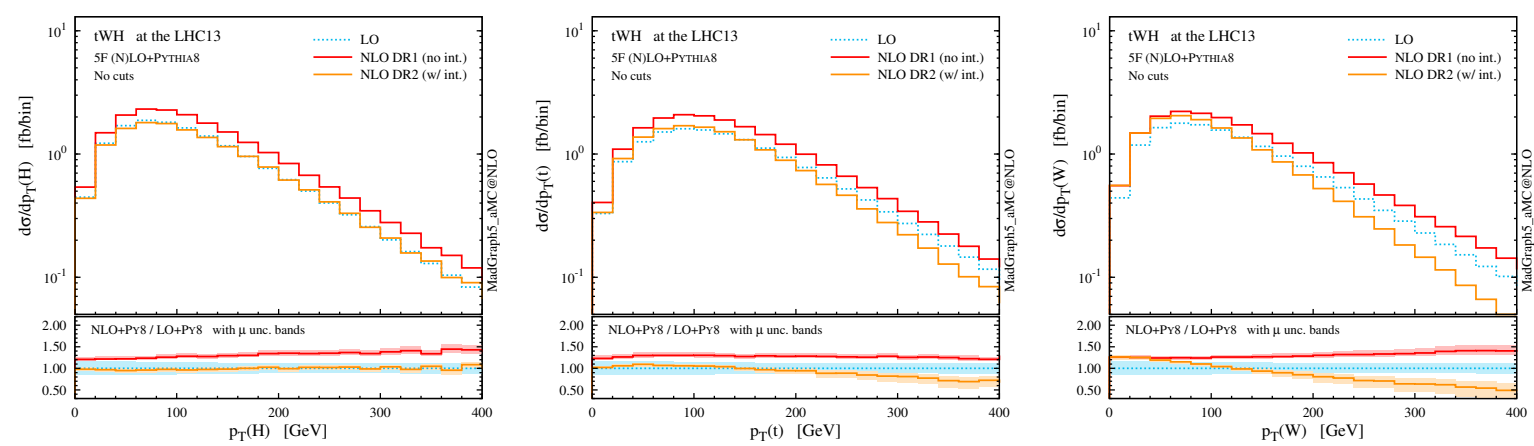

Figure 2: Transverse momentum distributions of the Higgs boson (left), the top quark (center) and the $W$ boson (right) at NLO+PYTHIA8.

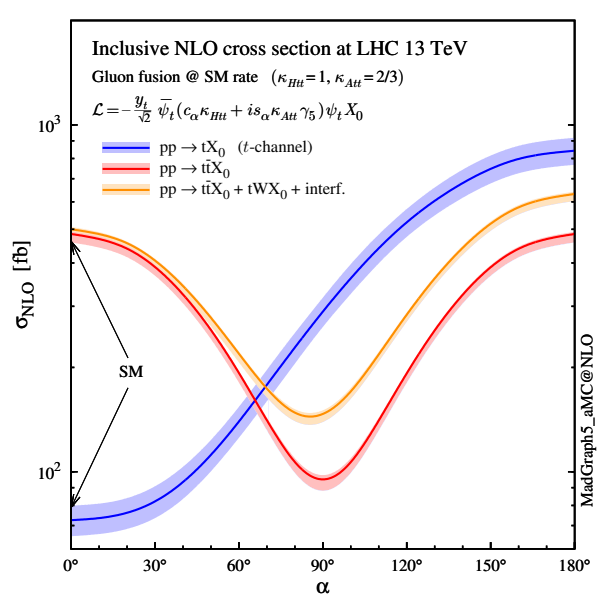

Figure 3: Total cross section with scale uncertainty for $t$-channel $t X_{0}$ (blue), $t t X_{0}$ (red) and $t t X_{0}+t W X_{0}$ production computed with DR2 (orange), as a function of the CP-mixing angle $\alpha$ in the HC Lagrangian. The point $\alpha=0^{\circ}$ corresponds to the SM scenario, while $\alpha=180^{\circ}$ corresponds to $y_{t}=-y_{t}(\mathrm{SM})$. 
Finally, we go beyond the Standard Model employing the Higgs Characterisation model $\left(H \rightarrow X_{0}\right)$, where the Higgs-top Yukawa interaction is described by the Lagrangian

$$
\mathscr{L}_{0}^{t}=-\bar{\psi}_{t}\left(c_{\alpha} \kappa_{H t t} g_{H t t}+i s_{\alpha} \kappa_{A t t} g_{A t t} \gamma_{5}\right) \psi_{t} X_{0}
$$

The SM case is reproduced when $c_{\alpha} \equiv \cos \alpha=1$ and $\kappa_{H t t}=1$. The sensitivity of the $t W X_{0}$ rate to the CP-mixing angle $\alpha$ is presented in fig. 3, together with $t t X_{0}$ and $t$-channel $t X_{0}$ for reference.

Acknowledgements: We are grateful to F. Maltoni, K. Mawatari and M. Zaro for their collaboration on this work. FD's participation in the workshop was supported by the IISN "MadGraph" convention 4.4511.10 and the IISN "Fundamental interactions" convention 4.4517.08.

\section{References}

[1] F. Demartin, F. Maltoni, K. Mawatari, and M. Zaro, Higgs production in association with a single top quark at the LHC, Eur. Phys. J. C75 (2015), no. 6 267, [arXiv: 1504.0061 ].

[2] S. Frixione, E. Laenen, P. Motylinski, B. R. Webber, and C. D. White, Single-top hadroproduction in association with a W boson, JHEP 07 (2008) 029, [arXiv: 0805.3067 ].

[3] E. Re, Single-top Wt-channel production matched with parton showers using the POWHEG method, Eur. Phys. J. C71 (2011) 1547, [arXiv:1009.2450].

[4] W. Beenakker, R. Hopker, M. Spira, and P. M. Zerwas, Squark and gluino production at hadron colliders, Nucl. Phys. B492 (1997) 51-103, [hep-ph/9610490].

[5] W. Hollik, J. M. Lindert, and D. Pagani, NLO corrections to squark-squark production and decay at the LHC, JHEP 03 (2013) 139, [arXiv:1207.1071].

[6] D. Goncalves-Netto, D. Lopez-Val, K. Mawatari, T. Plehn, and I. Wigmore, Automated Squark and Gluino Production to Next-to-Leading Order, Phys. Rev. D87 (2013), no. 1014002, [arXiv:1211.0286].

[7] R. Gavin, C. Hangst, M. Kramer, M. Muhlleitner, M. Pellen, E. Popenda, and M. Spira, Matching Squark Pair Production at NLO with Parton Showers, JHEP 10 (2013) 187, [arXiv: 1305.4061 ].

[8] A. Denner and R. Feger, NLO QCD corrections to off-shell top-antitop production with leptonic decays in association with a Higgs boson at the LHC, arXiv:1506.0744.

[9] J. Alwall, R. Frederix, S. Frixione, V. Hirschi, F. Maltoni, et al., The automated computation of tree-level and next-to-leading order differential cross sections, and their matching to parton shower simulations, JHEP 1407 (2014) 079, [arXiv: 1405.0301 ]. 